tung der Rassenkreuzung als solicher für die Konstitution oder für einzelne bestimmte Erkrankungen. Ob wirklich die Kreuzung von mongolider und nordischer Rasse in Skandinavien stärker, zu Tubexkulose prädestiniert? (Lundborg $)^{10}$ ). Und kann man wirklich Lmweitwirkung ausschließen and Kreuzung als solohe als den Grunid auffassen für disharmonische Körper- und Geistesanlagen gew isser Mischlinge? $\left.(\text { Mjoen })^{17}\right)$.

Eize Menge neuer Probleme stehen auf - an Arbeit, befruchtet von Mendels genialem Gedanken, wird es nicht fehlen. Jedenfalls hat er uns auch auf dem Gebiet der Erforschung des Menschen einen gewaltigen Schritt vorwärts gebracht

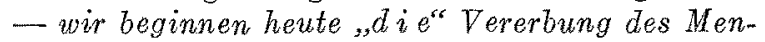
schen wirlelich etwas hennen zu lernen!

DaB diese Vertiefung und Erweiterung unserer Kenntnisse von der Vererbung beim Menschen deren praktische Anwendbarkeit in ganz anderem Licht erscheinen läBt, ist ganz selbstverständlich. So muBte die Rassenhygiene dadurch mächtig gefördert werden. - leider in unserem eigenen Vaterlanid nur theoretisch! Schweden hat das erste staatiche Institut für Rassenhygiene geschaffen unter der vielversprechenden Leitung Lundborgs ${ }^{18}$ ) - Norwegen besitzt das von Mjöen geschaffene Laboratorium, England und Amerika haben ältere, jetzt aber erneut erweiterte ,eugenische" Anstalten, Ungarn hat eine solche, die Schweiz kürzlich eine grobe Geldstiftung für diese Zwecke erhalten - Deutsohland hat auf diesem Gebiete gar nichts!

Wenn wir heute freudig feststellen, wie die Mendelforschung auch für das Studium der menschlichen Biologie, für die gesamte Medizin Neves und Ungeahntes brachte, wie sie besonders die für die Zukunft der Kulturvölker so anendlich wichtige, ja mit ausschlaggebende Rassenhygiene fördert und noch mehr fördern könnte, wenn entsprechende Arbeitsstätten bestünden, dürfen 'wir vielleicht an das Mendelgedenkjahr die Hoffnung, knüpfen, daß auch in Deutschland eine Forschungsanstalt für Rassenhygiene gegründet werde - das schönste Denkmal für Mendels Erblichkeitslehre!

\title{
Die Bedeutung der Mendelschen Gesetze für die Pflanzenzüchtung.
}

\author{
Von E. Baur, Berïin.
}

Für die Pllanzenzüchtung bedeutet die Entdeckung der Spaltungsgesetze den Ubergang ron rein empirischer Selektion" zu zielbew ußtem synthetischem Arbeiten. Man kann ruhig sagen, daß die ganze nächste Zukunft der Pflanzenzüchtung fast ausschließlich auf dem Ausbau der Kombinationszüchtung, d. h. auf nichts anderem als auf der unmittelbaren Umsetzung der Mendelschen Gesetze in die Praxis beruht.

Bei den Kulturpflanzen, die ganz oder doch stark vorwiegend Selbstbefruchter sind, führt jede richtig gehandhabte Auslese, die man heute stets als Individualauslese mit Bewertung des Individuums wach seiner Nachkommenschaft durchführt, dahin, dab fast reine und sehr weitgehend homozygotische Stämme erhalten werd'en, die dann durch weitere Selektion nur noch äuBerst langsam verbesiserungsfähig sind.

Große züchterische Fortschritte sind bei allen diesen Kulturpflanzen, zù denen z. B. Weizen, Gerste, Hafer, Erbsen und Bohnen gehören, nur dadurch möglich, dap man versucht, auf dem Wege der Kreuzungen gewisse vorteilhafte Eigenschaften, die in verschiedenen Sorten getrennt vorkommen, in einer Sorte zu vereinigen. Das glänzendste Beispiel hierfür sind Nilsson-Ehtes erfolgreiche Bemühungen, die Winterfestigkeit

16) Lundborg, Rassen- und Gesellschaftsprobleme in genetischer und meddizinischer Belesuchtung. "Hereditas"' (Lund) $I, 1920$.

17) Mjöen, Harmonisehe und wnharmonisehe Krenzungen. Zeitschr. f. Ethnol. 1921: der wenig ertragreichen schwedischen Landweizen mit den guten Eigenschaften der westeuropäischen hoch ertragreichen aber nicht winterfesten Dickkopfweizen $\mathrm{zu}$ vereinigen.

Den Weg für solche Vereinigung einzelner Eigenschaften hat Mendel gezeigt: in der zweiten Bastardgeneration, aus der Kreuzung der beiden genannten Rassen, müssen theoretisch Formen auftreten, welche die gewünschte Eigenschaftskombination verkörpern.

So leicht theoretisch eine solche Eigenschaftkombination ist, so schwer ist sie freilich in der Praxis, und zwar deshalb, weil bei diesen Kreuzungen sehr viel verschiedene Erbfaktoren mitspielen. Hoher Ertrag und Winterfestigkeit beruben jeweils für sich schon auf einer Kombination einer groBen Zahl von Erbfaktoren, und die Wahrscheinlichkeit ist sehr gering, daf in der $F_{2}$-Generation nun unter den vielen bunderttausend Kombinationen, die hier auftreten können $_{4}$ gerade diejenigen gefunden werden, welche man haben möchtè.

Eine solche Kombinationszüchtung setzt also voraus, dab oft lange Jahre hindurch sehr grobe $F_{2}$-Generationen (10-50000 Pflanzen) herangezogen und durchmustert werden.

Erfüllt man diese Bedingung, so bleiben auch die Erfolge nicht aus. Es ist bekannt, daß.z. B:

18) Anderson, The Swedish State-Institute for RaceBiological Investigation. Stockholm 1921. 
Nitsson-Ehle die gewünschte Kombination: - für Südschweden genügende Winterfestigkeit + hoher Ertrag - fast völlig erreicht hat, und daB die so gewonnenen neuen Kombinationen in Schweden sowohl gegenüber dem Landweizen wie gegenüber dem englischen Dickkopfweizen einen Mehrertrag von $40-50 \%$ geben.

Auf diesem Wege der Kombinationsztichtung sind noch sehr wesentliche Verbesserungen auch unserer deutschen Sorten erreichbar.

Auch bei uns spielt, für Weizen und Wintergerste besonders, die Winterfestigkeit eine sehr große Rolle. Weizen z. B., allerdings sehr ertragsarme Weizen, welche auch die schwersten Winter glatt überstehen und mindestens so winterfest sind wie Roggen, gibt es, und damit ist die Kombinationsaufgabe: ,Vereinigung der unbedingten Winterfestigkeit mit dem hohen Ertrag unserer westeuropäischen besten Dickkopf weizen" gegeben. Es ist nur eine Frage der Zeit, wann sie gelöst sein wird. Gerade solche strenge Winter wie der letzt vergangene, in dem große Flächen von Winterweizen vernichtet worden sind, und wo für den gesamten Winterweizen eiu starker Ernteausfall bedingt wird, lassen die rasche Lösung dieser Aufgabe besonders wichtig erscheinen.

Wir werden ferner versuchen müssen, einzelne Sorten unserer Wintergetreide ohne Ertragsschwächung wesentlich frühreifer zu machen und sie dahin zu bringen, $d a B$ sie die Troakenperioden im Frühsommer besser überstehen. Auch das dürfte durch Kreuzungen mit osteuropäischen und asiatischen Sorten erreichbar sein, allerdings erst in jahrelanger Arbeit.

Kine weitere auf dem Wege der Kombinationszüchtung unbedingt lösbare Aufgabe ist die Verbesserung der Backfähigkeit des Weizenmehles.

Auch für die Züchtung der Fremdbefruchter unter unseren Kulturpflanzen ist die Methode der Kombinationszüchtung — die ja, wie vorhin schon gesagt, nichts anderes ist als die Umsetzung der Spaltungsgesetze in die Praxis - von der größten Tragweite. Hier findet zwar bei der gewöhrlichen Fortpflanzung schon eine fortwährencie Neukomibination statt, nahezu jedes Individuum ist stark heterozygotisch. Es ist also waöglich, einfach durch Auslese hier noch wertvolle neue Kombinationstypen zu findien und $\mathrm{zu}$ isolieren. Aber um gröbore Fortschritte zu erzielen, wird man auch hier zielbewubt kreuzen müssen. ich erinnere nur daran, was es für den deutschen Rebbau bedeutet, wenn es uns gelingt, durch eine Kreuzung unserer Reben mit meltau- und reblausimmunen amerikanischen Rebarten eine Kombination herauszufinden, welche diese beiden epwünschten Eigenschaften vereinigt mit den guten Beereneigenschaften unserer besten enropäischen Sorten. Ahnliche Aufgaben lieben sich für alle unsere Obstsorten in grober Zahl nennen. Das Gleiche gilt in fast noch höherem Grade fur unsere Blumen und Gemüse.

Der auf diesem Wege mögliche züchterische Fortschritt ist sehr groß. Für unsere Getreidearten dürfte eine etwa 30-40prozentige Steigerung des Düchschnittsertrages unbedingt erreichbar sein. Was das für unsere Volkswirtschaft bedeutet, brauche ich wohl nicht weiter auszuführen.

Wer selbst auf dem Gebiete der Kombinationszüchtung Erfahrung hat, wind sich des Eindrucks nicht erwehren können, daß ungefähr ebensoviel, wiè die züchterische Arbeit der letzten 5-6 Jahrtausende uns vorwärts gebracht hat, künftig auf dem von Mendel geöffneten Wege in einem Jahrhundert etwa erreicht werden kann.

Mendel selbst hat wohl dio Tragweite seiner Entdeckung aicht geahnt. Der erste, der auf die ungeheure Wichtigkeit der Mendelschen Gesetzo fur die Pflanzenzüchtung hinwies, war $E$. Tschermak. Die gröBten Erfolge in dieser Richtung hat bisher unstreitig Nitsson-Ehle erzielt. 